\title{
Portable Laser Speckle Imaging System for Perfusion Imaging of Human Hand
}

\author{
Yiguang Zhang ${ }^{1}$, Fan Zhang ${ }^{1}$, Zhixia $\mathrm{Wu}^{1}$, Weijie $\mathrm{Wu}^{2}$, and Peng Miao ${ }^{1, \text { a }}$ \\ ${ }^{1}$ School of Communication and Information Engineering, Shanghai University, Shanghai 200444, \\ China; \\ ${ }^{2}$ Nanjing University of Posts and Telecommunications, Nanjing 210023, China; \\ apengmiao@shu.edu.cn
}

Keywords: Laser speckle contract imaging, perfusion, portable system, rehabilitation.

\begin{abstract}
In this study, we developed a new portable hand perfusion imaging system for rehabilitation evaluation based on laser speckle contrast imaging (LSCI). The new system measured perfusion changes in hand surface quantitatively. The hardware of portable imaging system consists of compact optical and electrical devices and is controlled by a user-friendly software interface. The software enables real-time perfusion image display and data plot of multiple region of interest. The portable system is applied to monitor the changes of hand perfusion during post-occlusive reactive hyperaemia (PORH) test.
\end{abstract}

\section{Introduction}

As an optical method, laser speckle contrast imaging (LSCI) has been used in imaging the perfusion of body surface $[1,2]$. With respect to the computed tomography, magnetic resonance imaging, ultrasound imaging, LSCI has advantages of contrast-free, high temporal resolution and low-cost [3]. LSCI can be used in both basic research and clinical applications [4]. LSCI also has some limitations, i.e. limited imaging depth, and two-dimensional imaging [5]. So, LSCI only provides perfusion information of surface tissue, such as cerebral blood flow (CBF) [6] and the skin perfusion index. In addition, the large volume of imaging system, complex configuration, and the poor software design limit its applications as a clinical tool.

In this study, we developed a portable imaging system with hardware integration for perfusion imaging of human hand. The portable system consists of compact optical and electronical elements. The integrated hardware design makes the imaging operation simpler. The feature-rich software enables real-time display of perfusion images and data in region of interest (ROI) areas. The software also provides offline data analysis functions facilitating the clinical diagnosis. We applied the portable imaging system in quantitative assessment of hand perfusion during post-occlusive reactive hyperaemia (PORH) test.

\section{Laser Speckle Contrast Imaging Theory}

When irradiating rough surface using laser light, laser speckle patterns are obtained as the random interference fringes. If slight movements present in the imaging area, speckle patterns blink randomly. The speckle fluctuations are related to the speed of movements [7]. By analyzing the fluctuations of speckle pattern, laser speckle contrast imaging (LSCI) technique provides high resolution detection of blood perfusion in biological tissues [8]. The principle of LSCI is based on the exposure of imaging camera. For a higher perfusion region, the speckle is fluctuated faster, so the image captured by camera is blurred due to the intensity integration in the exposure time. In LSCI, contrast value $K$ is used to describe the degree of blurring. Here's the formula of contrast value $K$ [9]:

$$
K=\frac{\sigma}{I}
$$


Where $T$ is the exposure time, $\sigma$ and $I$ are the standard deviation and the mean value of the intensities in the speckle image.

In practice, there are temporal and spatial LSCI algorithms for calculation contrast value $K$ [10]. In temporal algorithm, after obtaining a few images, e.g. 50 images, the contrast of each pixel is calculated based on the mean and standard deviation of all 50 values. The spatial algorithm requires one image and contrast of each pixel is calculated is a spatial window (e.g. $7 \times 7$, centered at that pixel). Temporal algorithm has a high spatial resolution, while spatial algorithm provides a high temporal resolution. Recently, hybrid method combining spatial and temporal algorithms has been proposed to control resolution and SNR [11].

\section{Hardware}

Figure 1 is the module design of our portable hand perfusion imaging system. The portable imaging system contains: (1) near-infrared laser light (wavelength of 780nm; power 10mW; Model L780p010, Thorlabs); (2) optical diffuser of beam shaping providing uniform illumination; (3) CMOS camera (resolution $1280 \times 720$; the bit of depth 10bit; frame rate 30fps; Model LSCI-S1, Dolphin Shanghai Biological Technology Co., Ltd.) for recording speckle pictures; (4) USB3.0 interface for imaging control and data transfer by the USB cable connected to the computer; (5) power adapter (INPUT: 100-240AC, 50 / 60Hz; OUTPUT: DC + 5V, 2A; model AD3015, Made in China) providing power supply of the whole system. The shell of portable imaging system is made of acrylic material (32 cm long, $22 \mathrm{~cm}$ wide, $35 \mathrm{~cm}$ high), and the total weight is $2.9 \mathrm{~kg}$. A soft cushion is placed at the bottom of the shell box to keep hand steady.

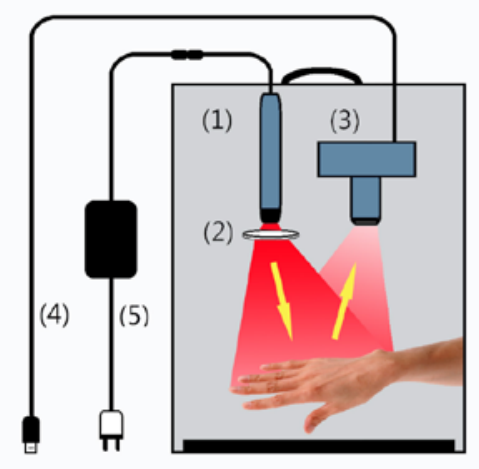

Fig. 1 Design diagram of portable hand perfusion imaging system: (1) near-infrared laser light; (2) optical diffuser; (3) CMOS camera; (4) USB cable; (5) power adapter.

\section{Software}

The software of portable imaging system provides imaging control and data management (Figure 2 (a)). The software was developed using the $\mathrm{C}++$ programming language in Microsoft Visual Studio 2010 platform. The software includes on-line experiment interface and off-line analysis interface, where the first interface is used for real-time imaging, display and data acquisition, the second interface is used for offline processing. The entire software consists of four modules: (1) imaging control module; (2) perfusion image display module; (3) ROI selection module; (4) data analysis module.

Imaging control module controls CMOS camera to capture raw speckle images. The perfusion image of human hand is calculated and displayed in real time using perfusion image display module. In order to maintain a high spatial and temporal resolution, we use the random process estimator algorithm [11] (the flow chart in Figure 2 (b)). At the same time, using ROI selection module, the user can select ROIs (Figure 3 (a)) and the corresponding perfusion index is plotted (Figure 3 (b)). This feature allows the clinicians to observe and compare dynamic changes of perfusion in different areas. During the data recording, user can press the P key in keyboard to mark events (Figure 3 (b)). 
(a)

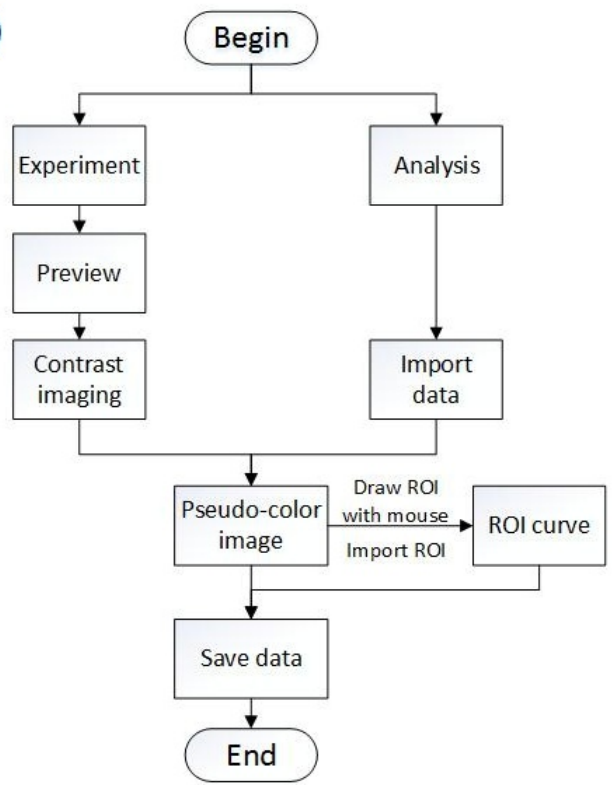

(b)

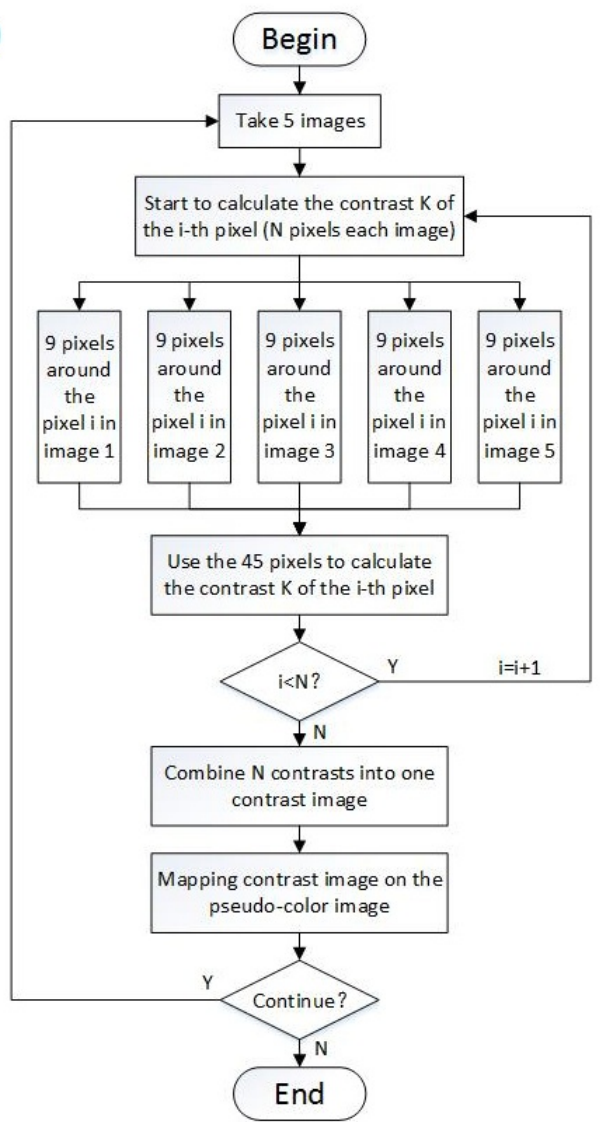

Fig. 2 (a) Software of portable imaging system; (b) Flow chart of perfusion image display module
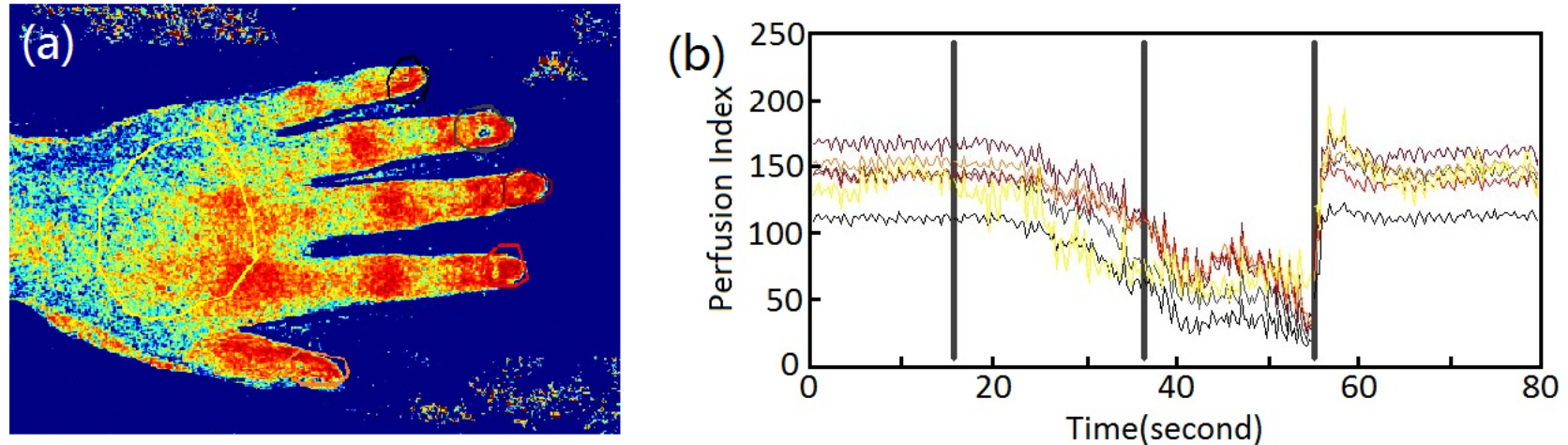

Fig. 3 (a) Hand perfusion image and selected ROIs; (b) Perfusion data of ROIs during the experiment.

\section{Monitoring hand perfusion during post-occlusive reactive hyperaemia (PORH) test}

The portable perfusion imaging system was applied to monitor the dynamic changes of hand perfusion during PORH test in experiments. In this study, five human subjects with normal blood pressure were imaged using the portable imaging system. An electronic sphygmomanometer was used to induce PORH by exerting pressure on the subject's upper arm cuff. To compare the perfusion index changes in different ROIs, we manually selected five fingertips and the back of hand as ROIs for investigation. Here is the protocol of the experiment:

(1) Wrap the belt to the subjects' arm cuff, put subject's hand into the portable imaging system;

(2) Select the ROIs in perfusion image display window; Start the recording of baseline state, press P key to mark the end of baseline, then start to exert pressure on the upper arm cuff using an electronic sphygmomanometer;

(3) Stop increasing the pressure when it reach $25 \mathrm{kPa}$, press the $\mathrm{P}$ key to mark it, press $\mathrm{P}$ key to mark the beginning of pressure release, and recording until the end of the monitoring.

(4) Removing subject's arm belt, turn off the software and device. 
Figure 3 (a) showed the hand perfusion image of one subject in the baseline recording procedure. Figure 3 (b) presented the perfusion data of all selected ROIs acquired in the whole experiment. In the baseline recording, the subjects were in the resting stage, so no significant changes were presented in perfusion index. Then we start to exert pressure on the upper arm cuff resulting in decrease of perfusion index. The decrease of perfusion index continued even when the pressure was kept at 25 $\mathrm{kPa}$. After release of the pressure, the perfusion index recovered rapidly, and the peak values were higher than baseline perfusion index. Finally, blood perfusion index returned to baseline level.

\section{Summary}

In this study, we developed a new portable hand perfusion imaging system based on laser speckle contrast imaging method. It enables real-time perfusion image display and data plot of multiple region of interest. The portable imaging system shows potentials in clinical rehabilitation evaluations.

\section{Acknowledgments}

This study was financially supported by the National Natural Science Foundation of China (61571284) and “Chen Guang” project by Shanghai Municipal Education Commission and Shanghai Education Development Foundation. Corresponding author: pengmiao@shu.edu.cn

\section{References}

[1] A.K. Dunn, Laser speckle contrast imaging of cerebral blood flow, Ann. Biomed. Eng., 40 (2012) 367-377.

[2] D.A. Boas, A.K. Dunn, Laser speckle contrast imaging in biomedical optics, J. Biomed. Opt., 15(2010) 011109.

[3] J. Senarathna, A. Rege, N. Li, N.V. Thakor, Laser Speckle Contrast Imaging: theory, instrumentation and applications, IEEE Rev. Biomed. Eng., 6(2013) 99-110.

[4] S.M.S Kazmi, L.M. Richards, C.J. Schrandt, M.A. Davis, A.K. Dunn, Expanding applications, accuracy, and interpretation of laser speckle contrast imaging of cerebral blood flow, J. Cereb. Blood Flow Metab., 35(2015)1076-1084.

[5] D. Briers, D.D. Duncan, E. Hirst, S.J. Kirkpatrick, M. Larsson, W. Steenbergen, T. Stromberg, O.B. Thompson, Laser speckle contrast imaging: theoretical and practical limitations, J. Biomed. Opt., 18(2013) 066018.

[6] C. Ayata, A.K. Dunn, Y. Gursoy-Özdemir, Z. Huang, D.A. Boas, M.A. Moskowitz, Laser speckle flowmetry for the study of cerebrovascular physiology in normal and ischemic mouse cortex, J. Cereb. Blood Flow Metab., 24(2004) 744-755.

[7] J.C. Dainty. Laser speckle and related phenomena, Berlin and New York, Springer-Verlag, 1975.

[8] J.D. Briers, S. Webster. Quasi real-time digital version of single-exposure speckle photography for full-field monitoring of velocity or flow fields. Opt. Commun., 116(1995) 36-42.

[9] J.W. Goodman. Speckle phenomena in optics: theory and applications. Roberts and Company Publishers, 2007.

[10]H. Cheng, Q. Luo, S. Zeng, S. Chen, J. Cen, H. Gong, Modified laser speckle imaging method with improved spatial resolution, J. Biomed. Opt., 8(2003) 559-564.

[11] P. Miao, N. Li, N.V. Thakor, S. Tong, Random process estimator for laser speckle imaging of cerebral blood flow, Opt. Express, 18(2010) 218-236. 